\title{
Application of Predictive Modelling to Improve the Discharge Process in Hospitals
}

\author{
Sayed Hisham ${ }^{1}$, Shahina Abdul Rasheed ${ }^{2}$, Brayal Dsouza ${ }^{2}$ \\ ${ }^{1}$ Healthcare Analytics, Baby Memorial Hospital, Kozhikode, India \\ ${ }^{2}$ Prasanna School of Public Health, Manipal Academy of Higher Education, Manipal, India
}

Objectives: To find out the factors influencing discharge process turnaround time (TAT) and to accurately predict the discharge process TAT. Methods: The discharge process of cardiology department inpatients in a tertiary care hospital was mapped over a month. The likely factors influencing discharge TAT were tested for significance by ANOVA. Multiple linear regression (MLR) was used to predict the TAT. The sample was divided into testing and training sets for regression. A model was generated using the training set and compared with the testing set for accuracy. Results: After a process map was plotted, the significant factors influencing the TAT were identified to be the treating doctor, and pending evaluations on the day of discharge. The MLR model was developed with Python libraries based on the two factors identified. The model predicted the discharge TAT with a $69 \% \mathrm{R}^{2}$ value and 32.4 minutes (standard error) on the testing set and a $77.3 \% \mathrm{R}^{2}$ value and 26.7 minutes (standard error) on the overall sample. Conclusions: This study was an initiation to find out factors influencing discharge TAT and how those factors can be used to predict discharge in the hospital of interest. The study was validated and predicted the TAT with 77\% accuracy after the significant factors that affect the discharge process were identified.

Keywords: Machine Learning, Regression Analysis, Linear Model, Patient Discharge

\section{Introduction}

The discharge process is a routine feature in any hospital that takes care of inpatients [1]. The discharge process com-

Submitted: February 12, 2020

Revised: 1st, April 24, 2020; 2nd, June 11, 2020; 3rd, July 1, 2020

Accepted: July 21, 2020

\section{Corresponding Author}

Brayal Dsouza

Prasanna School of Public Health, Manipal Academy of Higher Education, Manipal 576104, India. Tel: +91-99004-05393, E-mail: brayal.dsouza@manipal.edu (http://orcid.org/0000-0002-81539694)

This is an Open Access article distributed under the terms of the Creative Commons Attribution Non-Commercial License (http://creativecommons.org/licenses/by$\mathrm{nc} / 4.0 /$ ) which permits unrestricted non-commercial use, distribution, and reproduction in any medium, provided the original work is properly cited.

(c) 2020 The Korean Society of Medical Informatics mences with the treating physician approves the termination of an inpatient course of care. Generally, the process involves the physician informing the patient that he or she will be discharged, preparation of the discharge summary, and bill settlement, after which the patient can leave the hospital. The turnaround time (TAT) for this discharge process covers the time from when the treating physician initiates the discharge until the patient leaves the hospital. Often the discharge process tends to take several hours because of many factors. The time taken for completion of the discharge process maybe extended by bottlenecks due to various factors, such as the number of steps involved in the process, the time taken for the completion of these steps, and interruptions that may hinder the flow of the process. It would be beneficial for hospitals as well as patients to anticipate such time consumption.

The discharge TAT and delays in discharging patients are a long-standing challenge of hospitals [2]. Delays in a hospi- 


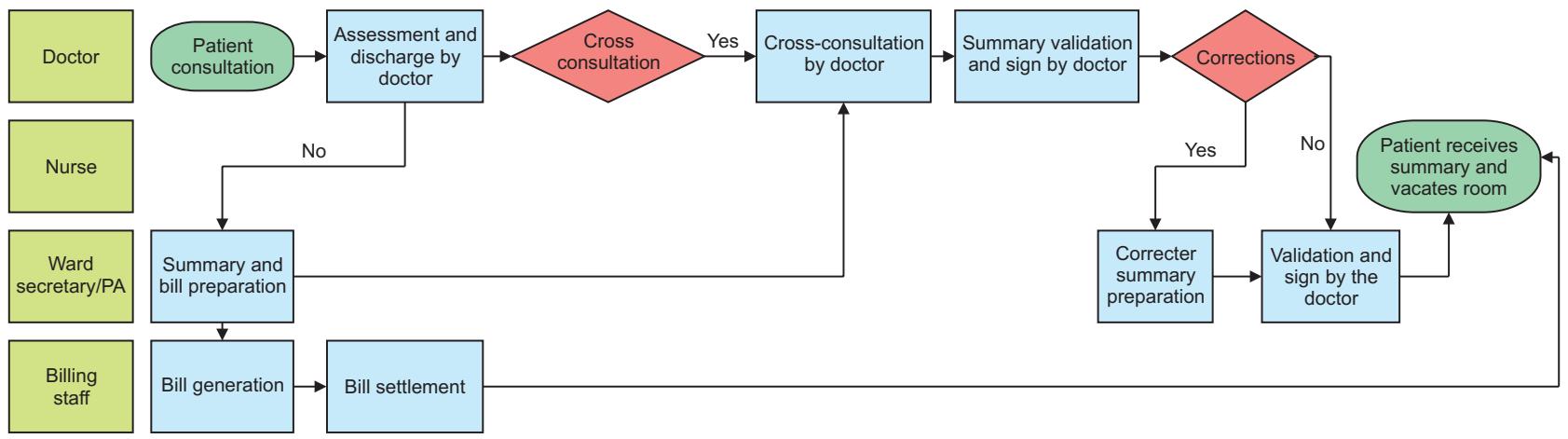

Figure 1. Discharge process.

tal's discharge process lead to further delays in other hospital functions, such as admissions, bed allocations, and transfer of patients. This leads to adverse effects on the reputation of the hospital [3]. Such delays cause overcrowding in admission queues and emergency departments of the hospital as well as ambulance diversions. This can be considered an opportunity loss for the hospital [4].

Having an estimated time given for the discharge process may help the hospital and all the healthcare providers involved in patient care streamline the discharge process and explore further ways to improve hospital services. This study aimed to identify the various features influencing discharge TAT and predict it through machine learning.

The discharge process usually involves two main stages, first, informing the patient that he or she will be discharged to final summary preparation, and second, bill initiation and clearance $[1,5]$. The hospital considered in this research was no different. This process involves many activities and many pending tasks, such as last-day investigations and cross-consultations leading to unanticipated and unpredictable delays [6]. The process involved in the study is presented in Figure 1 .

In this study, we developed a method for predicting the discharge TAT in a hospital. With this method of TAT prediction, further opportunities to improve the quality of services offered can be explored in the hospital. The proposed method uses multiple linear regression (MLR), which has not been widely explored in the literature.

We focused on addressing operational inefficiencies in handling the administrative processes involved in hospital discharges. Successfully predicting the discharge TAT could help with streamlining various administrative functions of the hospital. Knowing the TAT for each discharge process can help administrators and other staff members anticipate, plan, and execute other dependent functions, such as housekeeping, maintenance, and sterilization of inpatient rooms for the next admission, resulting in a seamless discharge-admission cycle for inpatient beds. Thus, it would be possible to avoid the mismanagement of the aforementioned functions.

This study can provide insight into using machine learning to predict the discharge processing time and its various applications in administrative functions such as planning, resource allocation, and so forth. Therefore, this study can be used as a reference for further studies further exploring various predictive models for estimating discharge TAT and its applications in healthcare settings.

\section{Methods}

This study was carried out in the cardiology department of a tertiary care hospital based on data collection and observations of all the discharges spanning over a month. This study was completed within 3 months from September to November of 2019.

\section{Outcome}

The study was divided into two parts.

Part 1: We observed the process-flow of the discharge and then noted the time consumed by each activity. A chart for noting down each activity in the process was prepared, and time taken for each activity was recorded for 108 discharges in the hospital over the course of 1 month. The activities identified are presented in Figure 2. The various factors influencing discharge TAT were identified.

Part 2: The anticipated discharge TAT for each patient was predicted based on the influencing factors identified in Part 1. The time taken for each activity was noted down as shown in Table 1. 


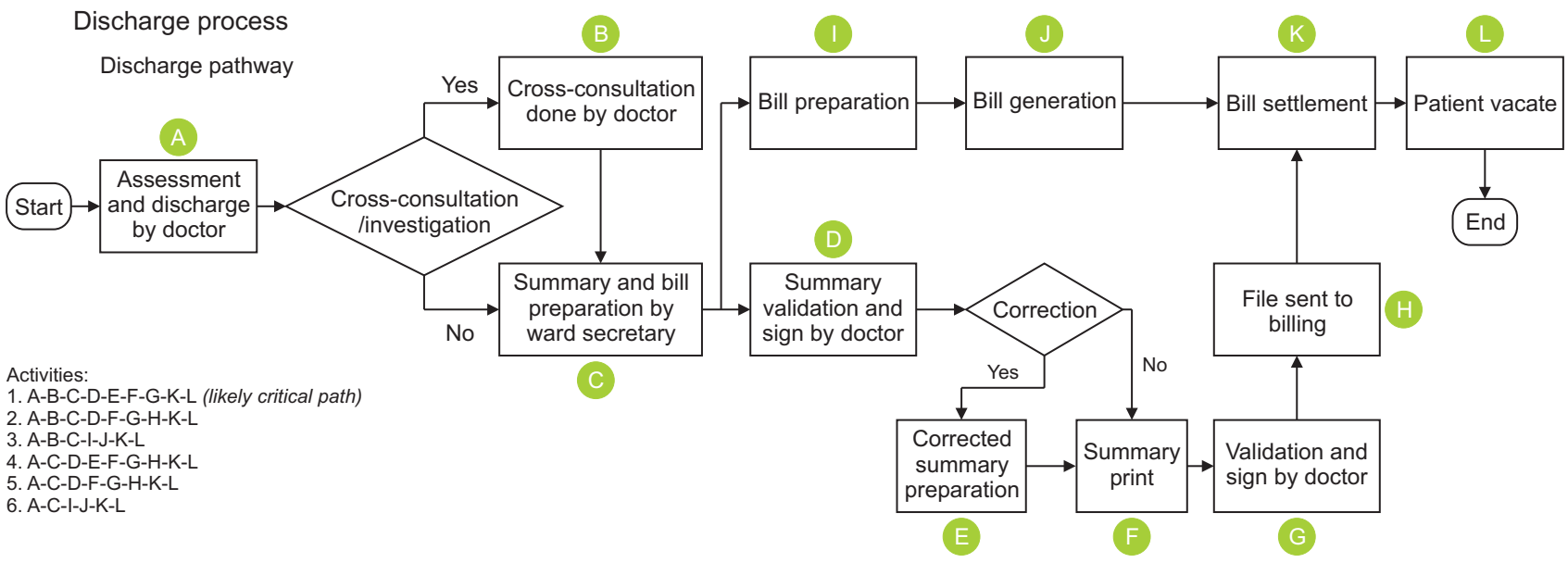

Figure 2. All the pathways in the discharge process.

Table 1. Activity code in Figure 2 and corresponding activities

\begin{tabular}{cl}
\hline $\begin{array}{c}\text { Activity } \\
\text { code }\end{array}$ & \multicolumn{1}{c}{ Activity } \\
\hline A-B & Assessment to Cross-consultation (if any) \\
B-C & Cross-consultation to Summary and bill preparation \\
A-C & Assessment to Summary preparation \\
C-D & Summary preparation to Validation by treating doctor \\
D-E & Validation and correction by doctor \\
E-F & Corrected summary preparation and printing \\
D-F & Summary validation and printing (if no corrections) \\
F-G & Summary printing to Final validation \\
G-H & Validated summary moved for billing \\
C-I & Bill summary preparation to Bill preparation \\
I-J & Bill preparation to Bill generation \\
H-K & File sent to billing after summary validation \\
J-K & Bill generation to Bill settlement \\
K-L & Bill settlement to Vacating of room by patient \\
\hline
\end{tabular}

\section{Data Collection}

Data was collected exhaustively for all 108 discharges that occurred in the span of 1 month in the cardiology department. The data was collected by tracking each discharge process and the time taken for its completion from the hospital's discharge tracking application.

The discharge process flow used for this study was based on the design created and maintained by the hospital in its standard operating procedure (SOP) documents. The discharge-process design explained above is followed throughout the hospital irrespective of the admitting department.

Part 1: The factors affecting the discharge process were identified through analysis of the discharge process flow.
The discharge process is identical and consistently followed throughout the various hospital departments with the aid of a checklist. The checklist includes the various steps of the discharge process (as shown in Figure 2, Table 1). Multiple combination of all these steps as factors were tried and analyzed to find out which of those steps contributed significantly to the discharge TAT. An ANOVA analysis was done to find out the factors contributing to the variance in time taken for discharge.

Part 2: For the creation of the predictive model and testing of the model, the data was randomly divided into training and testing sets using the Python sklearn library [7]. The scope for regression analysis was explored, and it revealed that the training set was normally distributed, and MLR was chosen for modelling. The residual analysis was done, and a model was prepared using MLR. Its accuracy was tested with the testing set of the data. The above-mentioned combinations produced a model with the best accuracy of $77 \%$. The analysis of the model can be seen in the Python-Jupyter Notebook of this study [7]. All the analysis and visualization was done using the sklearn, numpy, matplotlib, and pandas libraries of Python 3.6 [8].

\section{Results}

Part 1: All the factors that may affect the discharge process were identified by tracking each discharge based on the discharge process flow activities checklist used in the hospital. The various factors analyzed based on this checklist were the following: (1) Assessment, (2) Cross-consultation, (3) Summary preparation, (4) Validation by treating doctor, (5) Corrected summary preparation, (6) Summary printing, (7) Final validation, (8) Validated summary moved for billing, 
Table 2. Accuracy of the predicted model on the test-set

\begin{tabular}{|c|c|c|c|c|c|c|}
\hline \multirow{2}{*}{ SI no. } & \multirow{2}{*}{ Doc } & \multirow{2}{*}{ Pending tests } & \multicolumn{2}{|c|}{ TAT (min) } & \multirow{2}{*}{ Difference } & \multirow{2}{*}{ Squared difference } \\
\hline & & & Actual & Model & & \\
\hline 1 & Doc1 & Yes & 225 & 208.10743 & 16.89257 & 285.4 \\
\hline 2 & Doc1 & Yes & 210 & 208.10743 & 1.89257 & 3.6 \\
\hline 3 & Doc3 & No & 130 & 113.11635 & 16.88365 & 285.1 \\
\hline 4 & Doc3 & No & 105 & 113.11635 & -8.11635 & 65.9 \\
\hline 5 & Doc4 & No & 51 & 80.299866 & -29.29990 & 858.5 \\
\hline 6 & Doc3 & Yes & 233 & 191.25022 & 41.74978 & 1743.0 \\
\hline 7 & Doc1 & No & 122 & 129.97356 & -7.97356 & 63.6 \\
\hline 8 & Doc4 & Yes & 240 & 158.43373 & 81.56627 & 6653.1 \\
\hline 9 & Doc3 & Yes & 173 & 191.25022 & -18.25020 & 333.1 \\
\hline 10 & Doc3 & Yes & 234 & 191.25022 & 42.74978 & 1827.5 \\
\hline 11 & Doc1 & Yes & 179 & 208.10743 & -29.10740 & 847.2 \\
\hline 12 & Doc2 & No & 120 & 86.433066 & 33.56693 & 1126.7 \\
\hline 13 & Doc4 & Yes & 189 & 158.43373 & 30.56627 & 934.3 \\
\hline 14 & Doc3 & No & 173 & 113.11635 & 59.88365 & 3586.1 \\
\hline 15 & Doc4 & No & 51 & 80.299866 & -29.29990 & 858.5 \\
\hline 16 & Doc3 & Yes & 219 & 191.25022 & 27.74978 & 770.1 \\
\hline 17 & Doc1 & No & 120 & 129.97356 & -9.97356 & 99.5 \\
\hline 18 & Doc1 & Yes & 225 & 208.10743 & 16.89257 & 285.4 \\
\hline 19 & Doc1 & Yes & 205 & 208.10743 & -3.10743 & 9.7 \\
\hline 20 & Doc3 & Yes & 192 & 191.25022 & 0.749777 & 0.6 \\
\hline 21 & Doc6 & Yes & 142 & 189.06024 & -47.06020 & 2214.7 \\
\hline 22 & Doc1 & No & 132 & 129.97356 & 2.026439 & 4.1 \\
\hline MAE (min) & & & & & & 25.24 \\
\hline $\operatorname{MSE}^{\mathrm{a}}\left(\min ^{2}\right)$ & & & & & & 1038.88 \\
\hline RMSE (min) & & & & & & 32.23 \\
\hline $\mathrm{R}^{2}(\%)$ & & & & & & 69.2 \\
\hline $\mathrm{SE}(\min )$ & & & & & & 32.4 \\
\hline
\end{tabular}

MAE: mean absolute error, RMSE: root mean squared error, MSE: mean squared error, SE: standard error.

${ }^{a}$ Residual sum of square.

(9) Bill summary preparation, (10) Bill generation, (11) File sent to billing after summary validation, (12) Bill generation, (13) Bill settlement, and (14) Vacating of room by patient.

An ANOVA analysis of the data produced the following results.

- Factors 1, 3, and 7 in combination attributed to the "treating doctor": It was observed that the discharge TAT varied significantly with different treating doctors.

- Factor 2 attributed to "final day cross-consultation": A pending investigation or cross-consultation on the last day of discharge leads to significant delay in the discharge TAT (Table 2).
As seen in Table 3 and Figures 3-5, the significant factors influencing the discharge TAT were the treating doctor and pending cross-consultations. This contributed for over 30\% and $56 \%$ of the variation, respectively, as evidenced by the $\mathrm{R}^{2}$ value. Hence, these two factors were considered for developing the model for predicting discharge TAT.

Part 2: The sample consisted of 108 items. This was split into $80 \%$ training set and $20 \%$ testing set for the out-of-sample accuracy. Then, 86 items were randomly chosen from the sample using the Python numpy library. Using the sklearn library, the algorithm below was derived for predicting the 
Table 3. ANOVA analysis of identified factors

\begin{tabular}{|c|c|c|c|c|c|}
\hline \multirow{2}{*}{ Factor } & \multirow{2}{*}{ Count in the sample (n) } & \multicolumn{2}{|c|}{ TAT (min) } & \multirow{2}{*}{$p$-value } & \multirow{2}{*}{$\mathrm{R}^{2}(\%)$} \\
\hline & & Mean & SD & & \\
\hline Treating doctors & & & & 0.001 & 30.4 \\
\hline Doc1 & 28 & 167 & 46 & & \\
\hline Doc2 & 4 & 125 & 24.8 & & \\
\hline Doc3 & 36 & 158 & 44 & & \\
\hline Doc4 & 12 & 100 & 67 & & \\
\hline Doc5 & 8 & 62 & 48 & & \\
\hline Doc6 & 20 & 153 & 43 & & \\
\hline Billing & & & & 0.318 & 0.94 \\
\hline Insurance & 31 & 149 & 85 & & \\
\hline Self & 76 & 153 & 56 & & \\
\hline Pending evaluations & & & & 0.001 & 56.62 \\
\hline Yes & 53 & 187 & 37 & & \\
\hline No & 55 & 103 & 36 & & \\
\hline Total & & 144 & 55 & N/A & N/A \\
\hline
\end{tabular}

TAT: turnaround time, N/A: not applicable.

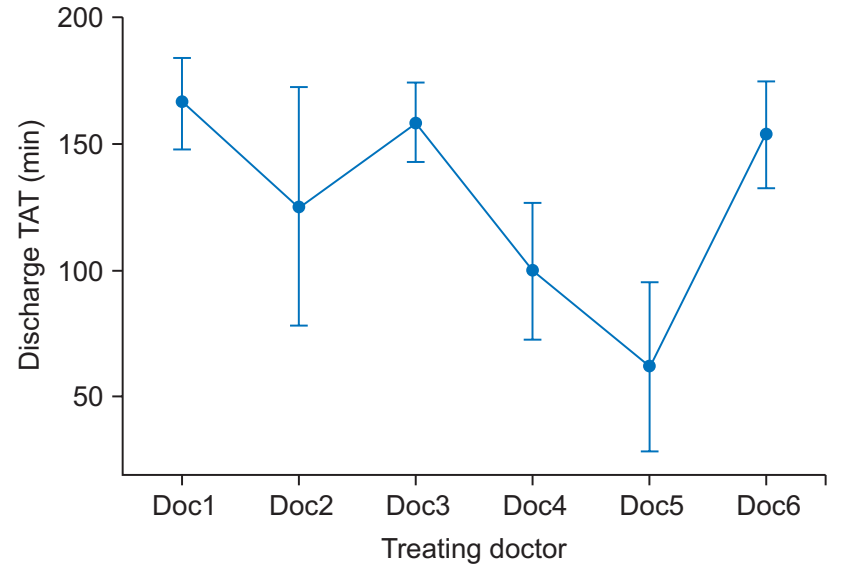

Figure 3. Interval plot discharge TAT vs. doctors. Bar represents as 95\% confidence interval for the mean. The pooled standard deviation was used to calculate the intervals. TAT: turnaround time.

discharge TAT:

Discharge TAT $=129.97+0.0$ Doc_Doc1 -43.5 Doc_Doc2 - 16.86 Doc_Doc3 - 49.67 Doc_Doc4 97.0 Doc_Doc5 - 19.05 Doc_Doc6 + 0.0 Pendingtests_No +78.13 Pendingtests_ yes

An MLR model was chosen for this study because, as seen in the residual plots in Figure 3, the residuals showed a fairly random pattern. The randomness in the residual plots, especially in the fitted plot, indicated that regression was the

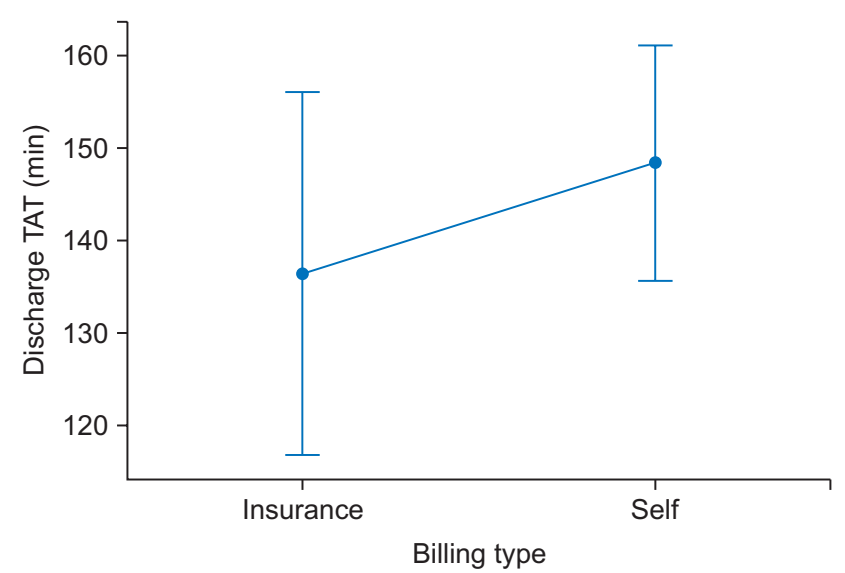

Figure 4. Interval plot discharge TAT vs. billing type. Bar represents as 95\% confidence interval for the mean. The pooled standard deviation was used to calculate the intervals. TAT: turnaround time.

ideal choice of algorithm.

The model was tested on the test set, and the following results were obtained. On evaluation, the model showed a mean squared error (MSE) of 1038.88 and an $\mathrm{R}^{2}$ value of $69.2 \%$. The out-of-sample accuracy is shown in Table 2. The residual plots for the model are shown in Figure 6. The model predicted values were compared with the actual data of the discharge TAT in the testing set, and the following results were obtained.

On evaluation with the complete set of 108 samples, the 
model predicted the TAT with an accuracy of $77.3 \%\left(\mathrm{R}^{2}\right.$ value) and an MSE of $702.26\left(\mathrm{~min}^{2}\right)$. The model showed a standard error of 26.7 minutes with a $95 \%$ confidence in-

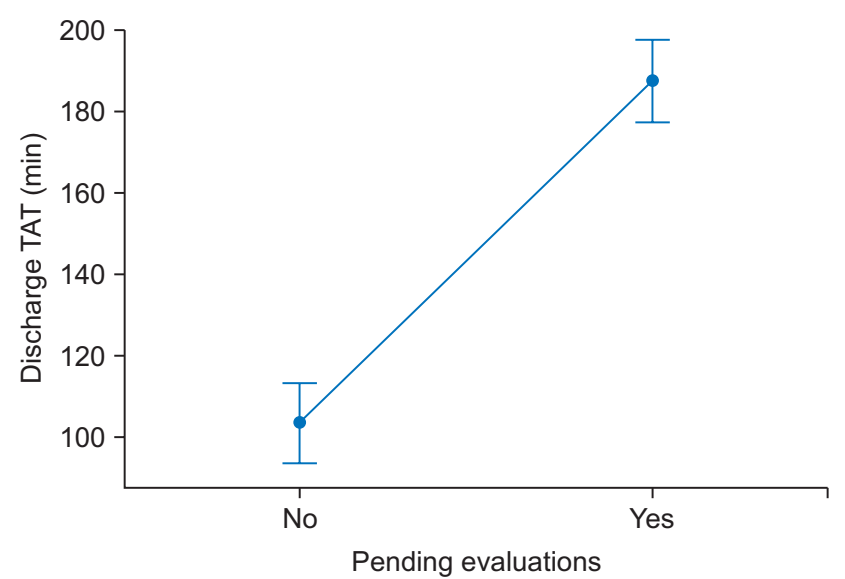

Figure 5. Interval plot discharge TAT vs. pending evaluations. Bar represents as 95\% confidence interval for the mean. The pooled standard deviation was used to calculate the intervals. TAT: turnaround time.
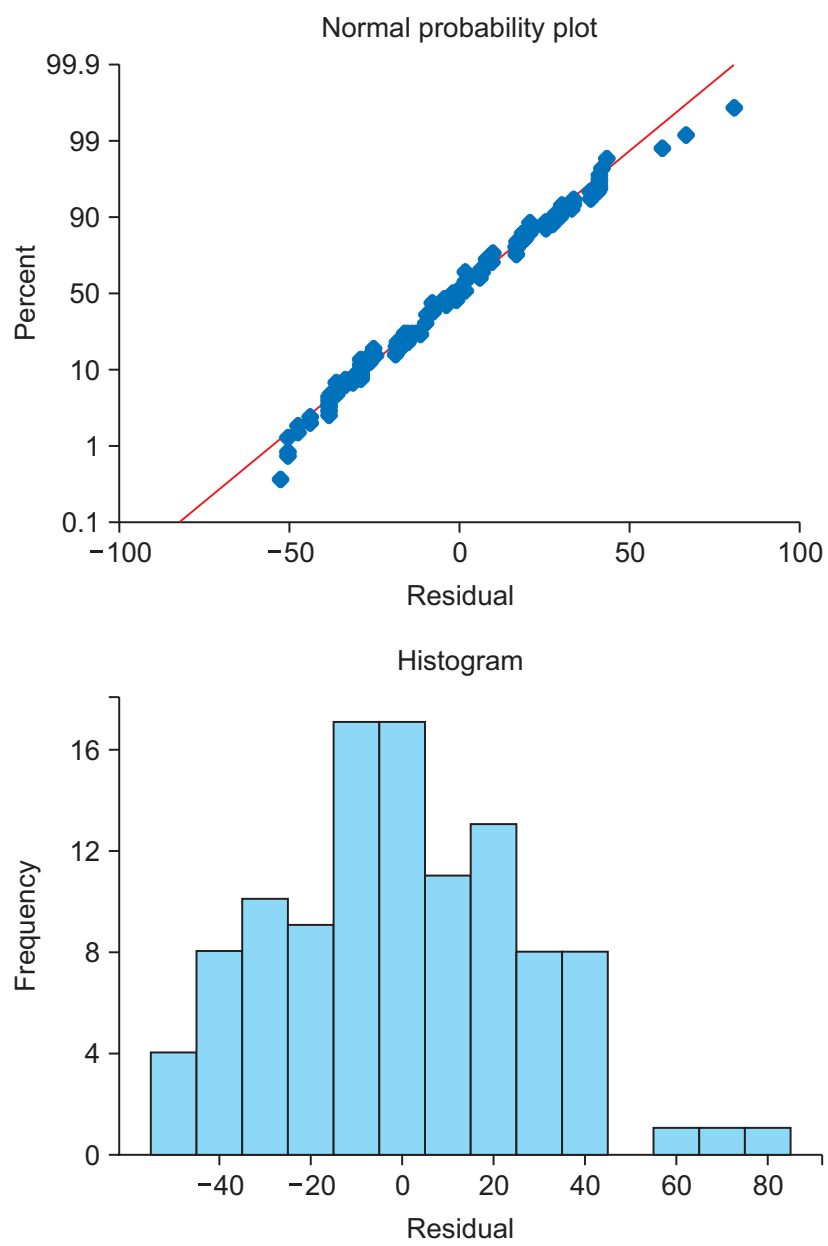

terval. This means that the model can predict the discharge TAT within an accuracy of \pm 52 minutes in $95 \%$ of the cases. The complete evaluation results of the overall sample are presented in Table 4.

\section{Discussion}

This study was carried out with the intention to predict the

Table 4. Evaluation of model on complete sample

\begin{tabular}{lc}
\hline Evaluation & Value \\
\hline MAE $(\min )$ & 20.86 \\
$\operatorname{MSE}^{\mathrm{a}}\left(\mathrm{min}^{2}\right)$ & 702.26 \\
$\mathrm{RMSE}(\mathrm{min})$ & 26.5 \\
$\mathrm{R}^{2}(\%)$ & 77.3 \\
$\mathrm{SE}(\min )$ & 26.7 \\
\hline
\end{tabular}

MAE: mean absolute error, RMSE: root mean squared error, MSE: mean squared error, SE: standard error.

${ }^{a}$ Residual sum of square.
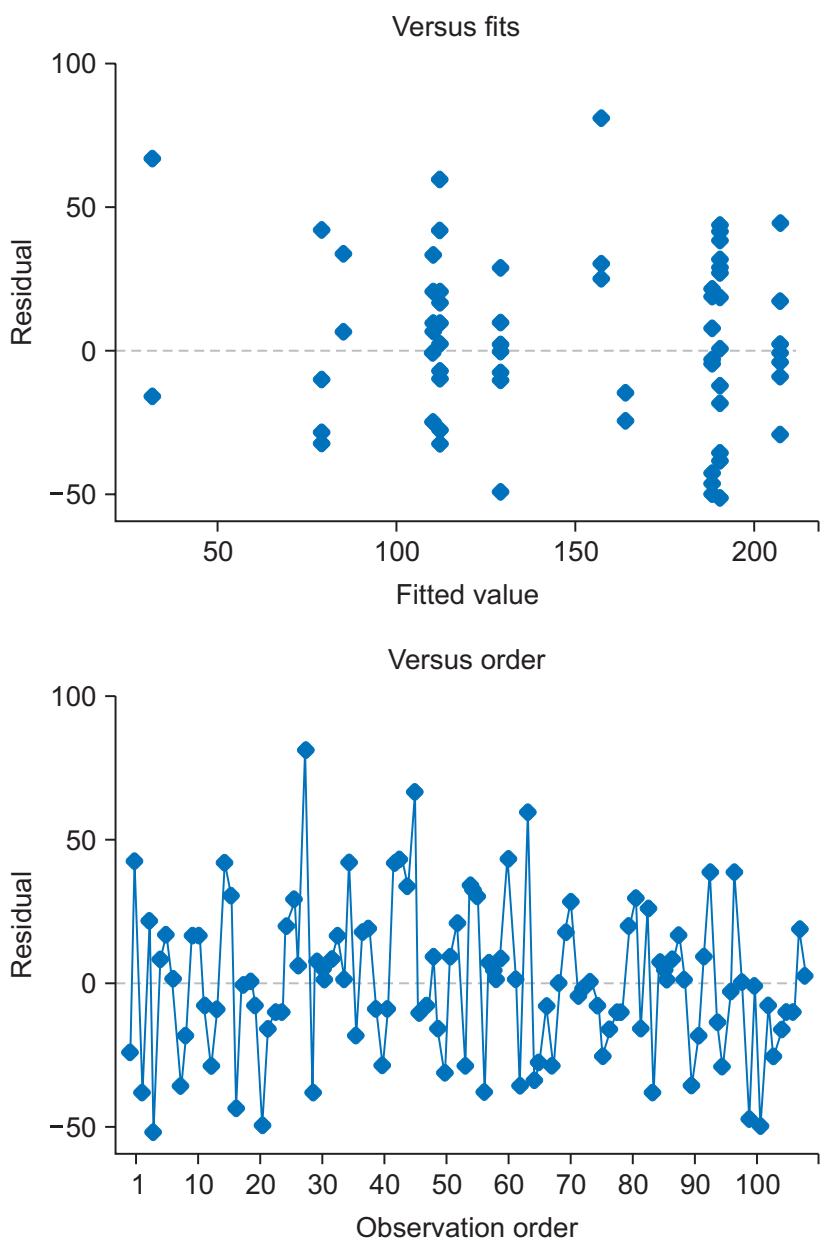

Figure 6. Residual plot for discharge turnaround time. 
discharge process TAT so as to coordinate better services for patients and patient's family or caregivers on the final day of their hospital stay, which will leave them with a lasting impression of the hospital. Although many studies have explored the benefits of predicting the time of discharge from the length-of-stay (LOS) point of view, very little research has been done on prediction of the TAT for the discharge process [9-11]. Consequently, the effectiveness and benefits of this type of prediction mechanism has not been studied in detail.

The model was based on MLR, which is a proven method for obtaining algorithms in analogous situations [12].

In a study titled, "The use of regression analysis to determine hospital payment: the case of Medicare's indirect teaching adjustment," by Thorpe [13], regression analysis was applied for its correct implementation in establishing reimbursement rates for hospitals under a government scheme.

In yet another study titled, "Predicting hospital length of stay using regression models: application to emergency department," by Combes et al. [9], the LOS of patients was estimated using linear regression. These models were validated and successfully applied to the classification and prediction of the LOS in the pediatric emergency department (PED) of the Lille Regional Hospital Center in France [9].

Several studies have compared the effectiveness of various algorithms for various use cases, such as "Residual analysis in regression" by StatTrek [14] and "A comparison of random forest regression and multiple linear regression for prediction in neuroscience," by Smith et al. [15]. Comparing the effectiveness of dozens of popular algorithms for predicting continuous data was beyond the scope of this study. This study focused on predicting the discharge TAT with reasonable accuracy. MLR was chosen for prediction based on residual analysis, and this produced a reasonably accurate result for practical implementation in the hospital.

Although this model had an accuracy of only $77 \%$, this can be attributed to the fact that fewer samples were collected due to time constraints and other limitations associated with manual observation of the process. Our subjective intuition is that the factors affecting the discharge TAT could certainly be the above-mentioned features in this set-up and that the method used is valid.

There are various examples of how linear regression has been used in the healthcare setting. A study titled "Improving the prediction of total surgical procedure time using linear regression modeling," [16] also used linear regression to create a predictive model to optimize service TAT in a clinical setting. In that study the linear regression model gave the most accurate value for the predicted procedure time. With a $77 \%$ accuracy for the predicted time, the result of that study is similar to the accuracy of the predicted TAT in this study. This can be used as an effective reference to use similar linear regression models for the prediction of TAT of similar administrative and clinical processes and procedures [16].

In a study by Bouphan and Srichan [17], a linear regression model was used to identify the factors affecting research to solve the health problems of health personnel in sub-district health promoting hospitals in Thailand. The study attempted to find the relationship between independent and dependent variables that could collectively predict the research. The model showed that there was a linear relationship between the dependent and independent variable. This helped in the creation of a model with a reasonable accuracy of $57 \%$, while the model developed in this study provided an accuracy of over $70 \%$.

The study by Freburger [18], analyzed the relationship between physical therapy services and the outcomes of patients with acute stroke. Although this study did not use a prediction model, it used MLR to evaluate the relationship. This demonstrates the versatility of the algorithm in helping identify significant relationships between factors with continuous variables.

Many studies on artificial intelligence and machine-learning research have focused on the clinical aspect of healthcare delivery [19]. For example, IBM Watson is a system that uses deep-learning, classification, and a regression algorithm to process unstructured data in medical records to identify patterns and predict outcomes $[19,20]$. The use of artificial intelligence is somewhat less potentially revolutionary in this domain as compared to patient care, but it can be used to substantially improve efficiency. This is needed in healthcare. For example, the average US nurse spends $25 \%$ of work time on regulatory and administrative activities [21]. However, in a hospital, there are also administrative departments, such as housekeeping, the front-office, engineering support services, IT support services, and so forth, that help them function efficiently. From a system perspective, a lot of resources, such as personnel, time, and infrastructure, are also devoted to this. This paper to address these administrative disciplines and application of machine-learning in improving administrative efficiency.

This is a rather novel approach, and very little previous research has considered using MLR to address and predict discharge TAT. The proposed MLR model for predicting discharge TAT is expected to be useful in managing other 
aspects of the discharge process, especially in terms of activities such as vacating hospital beds, disinfecting, and managing new admissions and the allocation of rooms for new inpatients.

This study was conducted to address the administrative hassles of delayed discharge processing. Only the time from the initiation of the discharge to the patient leaving the hospital bed was considered in this study. The time taken for clinical aspects of a patient's care was not considered because it was beyond the scope of this study. There is scope for further expanding this study to better understand any significant relationships between the prediction of discharge TAT and improved the quality of services in the hospital. This could not be investigated in this study due to limitations of time and resource constraints. Further studies and improved and larger datasets should be collected to enable better and more accurate prediction of any dependent variable. Therefore, it would be an invaluable service by healthcare analysts with such means and availability of data regarding the time taken for discharge to further develop this model.

\section{Conflict of Interest}

No potential conflict of interest relevant to this article was reported.

\section{ORCID}

Sayed Hisham (http://orcid.org/0000-0002-2806-4159)

Shahina Abdul Rasheed (http://orcid.org/0000-0002-0374-1387)

Brayal Dsouza (http://orcid.org/0000-0002-8153-9694)

\section{References}

1. Kaur H, Kochar R. A study on discharge process of discharged patients of a multispecialty hospital Ludhiana. Int J Eng Manag Res 2017;7(3):688-94.

2. Maloney CG, Wolfe D, Gesteland PH, Hales JW, Nkoy FL. A tool for improving patient discharge process and hospital communication practices: the "Patient Tracker". AMIA Annu Symp Proc 2007;2007:493-7.

3. Kripalani S, Jackson AT, Schnipper JL, Coleman EA. Promoting effective transitions of care at hospital discharge: a review of key issues for hospitalists. J Hosp Med 2007;2(5):314-23.

4. Falvo T, Grove L, Stachura R, Vega D, Stike R, Schlenker $\mathrm{M}$, et al. The opportunity loss of boarding admitted patients in the emergency department. Acad Emerg Med
2007;14(4):332-7.

5. Shukla K, Upadhyay S. Predictive modelling for turn around time (TAT) of discharge process for insured patients in a corporate hospital of Pune city. J Health Manag 2018;20(1):56-63.

6. Dalal AK, Poon EG, Karson AS, Gandhi TK, Roy CL. Lessons learned from implementation of a computerized application for pending tests at hospital discharge. J Hosp Med 2011;6(1):16-21.

7. Hisham S. Statistical analysis of discharge data [Internet]. [place unknown]: github.com; 2019 [cited at 2020 Jul 27]. Available from: https://github.com/hisham2k9/ Share_files/blob/master/dischage\%20model.ipynb.

8. McKinney W. Python for data analysis: data wrangling with Pandas, NumPy, and IPython. Sebastopol (CA): O'Reilly Media; 2012.

9. Combes C, Kadri F, Chaabane S. Predicting hospital length of stay using regression models: Application to emergency department. Proceedings of the 10th International Conference on Modeling, Optimization \& Simulation (MOSIM); 2014 Nov 5-7; Nancy, France.

10. Sullivan B, Ming D, Boggan JC, Schulteis RD, Thomas $S$, Choi J, et al. An evaluation of physician predictions of discharge on a general medicine service. J Hosp Med 2015;10(12):808-10.

11. De Grood A, Blades K, Pendharkar SR. A review of discharge-prediction processes in acute care hospitals. Healthc Policy 2016;12(2):105-15.

12. Pandis N. Multiple linear regression analysis. Am J Orthod Dentofacial Orthop 2016;149(4):581.

13. Thorpe KE. The use of regression analysis to determine hospital payment: the case of Medicare's indirect teaching adjustment. Inquiry 1988;25(2):219-31.

14. StatTrek. Residual analysis in regression [Internet]. [place unknown]: StatTrek; c2020 [cited at 2020 Jul 27]. Available from: https://stattrek.com/regression/residualanalysis.aspx\#.

15. Smith PF, Ganesh S, Liu P. A comparison of random forest regression and multiple linear regression for prediction in neuroscience. J Neurosci Methods 2013;220(1):85-91.

16. Edelman ER, van Kuijk SM, Hamaekers AE, de Korte MJ, van Merode GG, Buhre WF. Improving the prediction of total surgical procedure time using linear regression modeling. Front Med (Lausanne) 2017;4:85

17. Bouphan P, Srichan R. Factors affecting the research for solving health problem of health personnel at subdistrict health promoting hospitals. Procedia Soc Behav 
Sci 2017;237:1097-104.

18. Freburger JK. Analysis of the relationship between the utilization of physical therapy services and outcomes for patients with acute stroke. Phys Ther 1999;79(10):90618.

19. Stephen O, Sain M, Maduh UJ, Jeong DU. An efficient deep learning approach to pneumonia classification in healthcare. J Healthc Eng 2019;2019:4180949.
20. Kaymak S, Almezhghwi K, Shelag AA. Classification of diseases on chest X-rays using deep learning. In: Aliev R, Kacprzyk J, Pedrycz W, Jamshidi M, Sadikoglu F, editors. 13th International Conference on Theory and Applications of Fuzzy Systems and Soft Computing. Cham, Switzerland: Springer; 2018. p. 516-23.

21. Davenport T, Kalakota R. The potential for artificial intelligence in healthcare. Future Healthc J 2019;6(2):94-8. 